\title{
The development of fast and slow inferential responding: Evidence for a parallel development of rule-based and belief-based intuitions
}

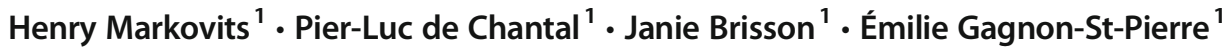

Published online: 15 April 2019

(C) The Psychonomic Society, Inc. 2019

\begin{abstract}
Dual process theories postulate the existence of two levels of processing, Type 1, which uses belief-based cues to make very rapid inferences, and Type 2, which uses more conscious, working memory-based processes that are, in principle, capable of making rulebased judgments. There is a common assumption that Type 1 processes are more rapidly produced, while Type 2 processes take more time. Evidence for this assumption is mixed. Recently, Newman, Gibb, and Thompson (Journal of Experimental Psychology: Learning, Memory, and Cognition, 43(7): 1154, 2017) have provided clear evidence that very fast responses use a combination of both belief-based and rule-based cues. In these studies, we initially replicated this basic result with adults using a different methodology and extended them to examine in greater detail patterns of change with restricted time. The results also show the same combination of rule-based and belief-based cues in fast responses, and suggest that time constraints differentially affect each form of cue. We then examined the developmental pattern of both fast and slow responding. Children aged between 8 and 10 years of age were examined using the methodology of the initial study. Results show that both developmental patterns and the effect of reduced time on children produce parallel changes in both belief-based and rule-based responding. These results suggest the existence of two simultaneously developing processes, consistent with a dual-strategy model of reasoning.
\end{abstract}

Keywords Reasoning $\cdot$ Development $\cdot$ Logical intuition $\cdot$ Dual strategy

\section{Introduction}

One of the more critical problems in the psychology of reasoning involves understanding the origins of non-logical versus logical responses. One of the more common general frameworks for understanding these different ways of making inferences is the dual-process model (De Neys \& Glumicic, 2008; Epstein, 1994; Evans \& Stanovich, 2013; Kahneman, 2011; Sloman, 1996; Stanovich, 1999). Although there are many different instantiations of this model, most suppose a distinction between processing systems that allow for rapid access to heuristic and belief-based associative information, which is generally responsible for non-logical responses (known as Type 1), and a slower working memory intensive system that is at least in principle capable of generating logical (rule-based) responses (known as Type 2). Generally, it is

Henry Markovits

henrymarkovits@gmail.com

1 Département de Psychologie, Université du Québec à Montréal, Montréal, Québec, Canada supposed that responses generated by Type 1 processes are more rapid than responses generated by Type 2 processes, which require effort and are under some form of conscious control. Although there might be exceptions to this, this suggests that rapid responses would use heuristic and/or beliefbased algorithms when making inferences, while slower responses would tend to be more logical, i.e., more rule-based. In a similar vein, this approach suggests that any reduction in available cognitive resources should reduce the capabilities of Type 2 processes, and correspondingly accentuate production of heuristic and belief-based responses.

There is in fact evidence that is consistent with this analysis. For example, when reasoning under time pressure (Evans \& Curtis-Holmes, 2005) or working memory load (De Neys, 2006), the tendency to use conclusion believability as an index of logical validity increases. Similarly, correlations between "logical" responses and measures of cognitive ability such as IQ (Stanovich, 1999) and the cognitive reflection test (Frederick, 2005; Toplak, West, \& Stanovich, 2011) have been interpreted as consistent with this basic analysis. In both cases, it is assumed that the Type 1 response is generated more rapidly and more easily and must subsequently be both recognized and inhibited by Type 2 processes when there is a 
conflict between the two. In a similar vein, the tendency for reasoners to generate an initial, heuristic response without putting this into doubt, known as cognitive miserliness (Stanovich \& Toplak, 2012; Toplak et al., 2011), depends on the same form of speed asymmetry.

On the other hand, there are theoretical and empirical arguments that suggest that this distinction may not be an adequate description of the difference between rapid and slow responding. One key component of dual-process models is the tension between non-logical and logical responses when these are produced in different time frames. However, there is a theoretical problem that must be resolved. This revolves around the problem of determining whether a rapid response generated by the Type 1 system is considered to be adequate or not with respect to the criteria used by the Type 2 system (De Neys, 2012). This implies that either the initial response is held in abeyance until a Type 2 response is produced (Evans, 2006), with any divergence between the two resolved at a later stage or some way of rapidly evaluating the initial response exists. If the former model was accurate, then one would expect that the time taken to produce a heuristic response that is in conflict with the logical response and the time taken to produce a heuristic response that was consistent with the logical response would be relatively similar. However, this does not seem to be the case at least for two commonly studied problems. The belief-bias effect refers to the tendency to judge a conclusion as logically valid when it is believable, irrespective of its true validity. Base-rate problems examine tendencies to judge the probability of sub-class of an event (e.g., the probability of having symptom $\mathrm{X}$ predicting having cancer) without considered the overall frequency of the class, i.e., the base rate (e.g., probability of having cancer). However, reaction time studies of the belief-bias effect and base-rate problems show that consistent heuristic responses are produced more rapidly than inconsistent heuristic responses, at least under certain conditions (De Neys \& Glumicic, 2008; Pennycook, Fugelsang, \& Koehler, 2012). This in turn suggests the existence of some form of logical intuition that allows a rapid evaluation of an initial response (De Neys, 2012).

Consistent with this idea of a form of logical intuition, there is evidence that people who are given instructions that explicitly require them to judge inferences on the basis of their beliefs are nonetheless sensitive to the conflict between these responses and the logical response (Handley, Newstead, \& Trippas, 2011; Morsanyi \& Handley, 2012). Similarly, studies that show that when people give heuristic responses that are in conflict with the logical response, a variety of internal cues indicate that they are unconsciously aware of the conflict (De Neys, 2012; De Neys, Vartanian, \& Goel, 2008; Simon, Lubin, Houdé, \& De Neys, 2015).

However, as pointed out by Newman, Gibb, and Thompson (2017), these results while suggestive remain indirect. For example, there is evidence that Type 2 responses are not inevitably logical but can contain elements that are consistent with Type 1 responses (Evans \& Stanovich, 2013; Quinn \& Markovits, 1998). Given the potential ambiguity of results that rely on conflict between rule-based and beliefbased responding, they suggest that a more direct way of examining the question is to look at the pattern of responses that people give when they are severely time constrained. Thus, Newman et al. (2017) used a dual-response paradigm in order to more directly investigate the characteristics of fast versus slow responding. This involves asking subjects to generate a sequential series of a very rapid initial response to a given problem and then to re-examine this response in a second phase where there is more time. Their results clearly indicate that under these circumstances, fast responses can reflect both non-logical (belief-based) and logical (rule-based) parameters. These results provide the strongest direct evidence that some form of logical intuition does indeed exist.

However, the fact that both fast and slow responses show the combined effect of both belief-based and rule-based criteria does raise some questions about the usefulness of dualprocess theories. As Newman et al. (2017) point out, if both fast and slow responding, which supposedly deploy Type 1 and Type 2 responding, differentially lead to similar combinations of responses, then the distinction between these dual processes may not be very useful. They suggest that other models might better represent this pattern of results. One such would be a single-process model (Kruglanski \& Gigerenzer, 2011; Osman, 2004) that uses complexity as a parameter to determine the relative contribution of rule-based and belief-based criteria. Another possible model would suggest that what appear to be similar responses might be produced by two different processes, such as that postulated by the dual-strategy model (Markovits, Brisson, \& de Chantal, 2017; Markovits, Brunet, Thompson, \& Brisson, 2013; Verschueren, Schaeken, \& d'Ydewalle, 2005). This model was originally proposed by Verschueren et al. (2005) as a way of integrating two opposing models of reasoning, mental model theory (Johnson-Laird \& Byrne, 1991, 2002), and probabilistic theories (Evans, Over, \& Handley, 2005; Oaksford \& Chater, 2003, 2007), and was described as another form of dual-process model. The more recent dual-strategy version supposes that reasoners have access to a statistical strategy, which uses rapid associative access to a wide range of information in order to generate an estimation of the likelihood of a putative conclusion (similar to the basic processes postulated by probabilistic theories) or to a counter-example strategy that uses a deliberate search for counter-examples based on an explicit representation of key elements (similar to the basic processes postulated by mental model theory, e.g., Johnson-Laird \& Byrne, 1994). For any given problem, people will use one or the other of these strategies, in contrast to dualprocess theories, which assume that Type 1 and Type 2 processes co-exist. In fact, recent research has shown that strategy use is an important individual difference that effects processing 
not only in reasoning tasks but also in very different domains (Markovits, Trémolière, \& Blanchette, 2018).

It remains, however, difficult to distinguish between these different models. One way of gaining some insight into which might better account for variability in reasoning would be to examine the developmental course of rule-based and beliefbased reasoning. In the following studies, we had two major goals. First, we attempted to replicate and extend the basic results of Newman and colleagues using a somewhat different methodology that can be applied to young children. Second, we examined the developmental pattern of fast and slow responding.

\section{Study 1a}

Although the dual-response paradigm has been used in previous studies with convincing results (Thompson, Prowse Turner, \& Pennycook, 2011), there remains a possibility that after the initial problem, the criteria used for slow responding might be incorporated into participants' fast responses. In this initial study, we attempt to replicate their basic findings using a series of inferences that must be resolved under very strict time constraints, followed by an equivalent series of inferences for which participants are given an unlimited time. The problems that we use involve two forms of conditional inferences, the Modus ponens inference (If P then Q, P is true) and the Affirmation of the consequent inference (If $\mathrm{P}$ then $\mathrm{Q}$, $\mathrm{Q}$ is true), and two equivalent forms of syllogistic inferences that follow the same logical pattern (all $\mathrm{X}$ are $\mathrm{A}, \mathrm{B}$ is $\mathrm{X}, \mathrm{P}$ is $\mathrm{A}$ ) and (all $\mathrm{X}$ are A. B is X, B is A). For convenience, we refer to these as Modus ponens and Affirmation of the consequent inferences. These were incorporated in order to reduce the possibility that participants would respond on the basis of a learned or rote response based solely on syntax. Studies have shown that the pattern of responses to these are similar, although the syllogistic version is somewhat developmentally earlier (Markovits, 2017). These inference forms were used since they are the simplest pair of inferences for which validity can be distinguished, which allows a useful developmental comparison.

In all cases, participants were presented with the invited conclusion, which they had to indicate as being valid or not. We also introduced a manipulation that varied the probability of the event or the category described in the conclusion, with half of the conclusions being more probable than the other half. This is somewhat similar to the manipulation used by Newman et al. (2017) in their second study.

The following examples illustrate how these items were constructed. For the Modus ponens inferences, we constructed pairs of premises with the same antecedent but with a consequent that was more or less probable. The following two inferences are examples of this:
Modus ponens High: If there is lightning, then there is a noise. There is lightning. Conclusion: there is a noise Modus ponens Low: If there is lightning, then there is a forest fire. There is lightning. Conclusion: there is a forest fire.

For the Affirmation of the consequent inferences, we constructed inferences with the same consequent, but with antecedents that were more or less probable, as in the following two examples:

Affirmation of the consequent High: If a ball is thrown into a window, the window will break. The window is broken. Conclusion: a ball was thrown into the window. Affirmation of the consequent Low: If a sofa is thrown into a window, the window will break. The window is broken. Conclusion: a sofa was thrown into the window.

Interpreting single responses as logical or not can result in a certain degree of ambiguity. Thus, this design was constructed to allow differences in response tendencies as indices of logical or non-logical tendencies. In order to do this, we used two separate difference scores. The extent to which participants are able to distinguish between the certainty of the Modus ponens inferences and the uncertainty of the Affirmation of the consequent inferences irrespective of the relative probability of the conclusion is measured by the difference between the acceptance rate of the Modus ponens inferences and the acceptance rate of the Affirmation of the consequent inferences. Similarly, the tendency to use relative probability as a criterion is measured by the rate of acceptance of the more probable conclusions minus the acceptance rate of the less probable conclusions.

In this first study, we examined a sample of adult participants. We presented an initial series of 16 inferences, of which half were Modus ponens inferences and half were Affirmation of the consequent inferences, with High and Low probable conclusions equally represented in both. Participants were given a very brief time to read and to respond to these inferences. Subsequently, a second series of 16 inferences that had the same characteristics as the initial series was presented, except that participants were given an unlimited time to respond. Our basic hypothesis was that consistent with the results observed by Newman et al. (2017), fast responses would show influences of both logic and relative probability.

\section{Method}

\section{Participants}

A total of 57 college-level students (28 females, 29 males; average age $=25$ years, 5 months) took part in this 
experiment. All participants were French-speaking students attending a French university in Montréal and were volunteers.

\section{Materials and design}

A computer program was created using Microsoft Visual Basic. The opening screen briefly described the study and asked the participant to indicate explicit acceptance to participate in addition to basic demographic information. Participants were then given a brief explanation of the inference tasks. They were told that they would be given three statements of which the last was a conclusion made on the basis of the first two and that their task was to indicate whether the conclusion was logical or not. Participants were given an example of a valid conclusion using a transitive relationship. They were then given an example of invalid conclusion also based on two relationships. They were then told that they would be given a brief time to respond either yes or no. They were also told that an indicator would turn red when they would have only $2 \mathrm{~s}$ to respond. In this initial Fast condition, participants were given $5 \mathrm{~s}$ for each inference.

After the initial series of 16 problems, participants were told that they would receive a second set of inferences, except that in this case they could take as much time as they wanted in order to be certain of their response.

The problem sets were constructed from an initial set of 32 inferences from each of which a high and low probability version was constructed. Two problem sets were constructed consisting of eight Modus ponens inferences and eight Affirmation of the consequent inferences, for which half of each form of inference corresponded to the high probability version of half the premise sets, while the other half corresponded to the low probability version of the other half of the premise sets (see Appendix). The two problem sets were chosen so that all the initial premises in each problem set were different. The two problem sets were randomly alternated between the fast and the slow conditions. For both the fast and the unlimited time conditions, the 16 problems were presented in an individually randomized order.

Thus, the number of conclusion acceptances was the dependent variable with Time (Fast, Unlimited) as a betweensubjects variable and Logical form (Modus ponens, Affirmation of the consequent) and Relative probability (High, Low) as within-subjects variables.

\section{Procedure}

Participants were seen individually in a quiet room and did the experiment on a portable computer.

\section{Results and discussion}

We first examined the rate at which participants failed to respond to the 16 inferences in the initial fast condition. Overall, $28.5 \%$ of the inferences resulted in a failure to respond. Any participant who did not respond to at least one of each of the four categories of inference was eliminated from subsequent analysis (there were two such participants). We then calculated the proportion of times that the Modus ponens high, the Modus ponens low, and the Affirmation of the consequent high, and the Affirmation of the consequent low inferences were accepted in both the fast and slow conditions (see Table 1).

We then performed an ANOVA with rate of conclusion acceptance as the dependent variable with Logical form (Modus ponens, Affirmation of the consequent), Relative Probability (High, Low) and Time (Fast, Unlimited) as repeated measures. This gave significant main effects of logical form, $\mathrm{F}(1,54)=134.86, \mathrm{p}<.001, \eta_{p}{ }^{2}=.714$, and relative probability, $\mathrm{F}(1,54)=72.76, \mathrm{p}<.001, \eta_{p}{ }^{2}=.574$, and significant interactions between time $\times$ logical form, $\mathrm{F}(1,54)=$ $71.27, \mathrm{p}<.001, \eta_{p}{ }^{2}=.569$, time $\times$ relative probability, $\mathrm{F}(1$, $54)=11.23, \mathrm{p}<.001, \eta_{p}{ }^{2}=.172$, and form $\times$ probability, $\mathrm{F}(1$, 54) $=6.04, \mathrm{p}<.02, \eta_{p}^{2}=.11$.

Post hoc analyses were done using the Tukey test with $\mathrm{p}=$ .05. Overall, there was a higher rate of acceptance of the Modus ponens $(\mathrm{M}=0.75, \mathrm{SD}=0.20)$ than of the Affirmation of the consequent inference $(\mathrm{M}=0.25, \mathrm{SD}=$ .20). Similarly, the overall acceptance rate was higher for the high probable $(\mathrm{M}=0.59, \mathrm{SD}=0.13)$ than for the low probable inferences $(\mathrm{M}=0.40, \mathrm{SD}=0.15)$. Analysis of the time $\times$ logical form interaction showed that the rate of acceptance of the Modus ponens inference was greater in the unlimited condition $(\mathrm{M}=0.84, \mathrm{SD}=0.21)$ than in the fast condition $(\mathrm{M}$ $=0.66, \mathrm{SD}=0.23$ ). The rate of acceptance of the Affirmation of the consequent inference was greater in the fast condition $(\mathrm{M}=0.36, \mathrm{SD}=0.26)$ than in the unlimited condition $(\mathrm{M}=$ $0.13, \mathrm{SD}=0.22$ ). Analysis of the time $\times$ relative probability interaction showed that the rate of acceptance of the high probable inferences was greater in the fast condition $(\mathrm{M}=$ $0.64, \mathrm{SD}=0.18)$ than in the unlimited condition $(\mathrm{M}=0.55$, $\mathrm{SD}=0.14)$. By contrast, the rate of acceptance of the low

Table 1 Proportion of conclusion acceptance (standard deviations in parentheses) as a function of Form (Modus ponens (MP), Affirmation of the consequent (AC)), and level of Relative probability (High, Low) as a function of allotted Time (Fast, Unlimited)

\begin{tabular}{llllll}
\hline Time & MP & & & AC \\
\cline { 2 - 3 } \cline { 5 - 6 } & High & Low & & High & Low \\
\hline Fast & $0.81(0.21)$ & $0.50(0.36)$ & $0.48(0.37)$ & $0.25(0.27)$ \\
Unlimited & $0.93(0.15)$ & $0.74(0.33)$ & $0.16(0.27)$ & $0.10(0.20)$ \\
\hline
\end{tabular}


probable inferences in the unlimited condition $(\mathrm{M}=0.43$, SD $=0.16$ ) was not significantly different than in the fast condition $(\mathrm{M}=0.38, \mathrm{SD}=0.23)$. Analysis of the logical form $\times$ relative probability interaction showed that the acceptance rate of the Modus ponens inference was higher when the conclusion was highly probable $(\mathrm{M}=0.87, \mathrm{SD}=0.15)$ than when the conclusion was less probable $(\mathrm{M}=0.62, \mathrm{SD}=0.29)$. Similarly, the acceptance rate of the Affirmation of the consequent inference was higher when the conclusion was high probable $(\mathrm{M}=0.31, \mathrm{SD}=0.27)$ that when the conclusion was low probable $(\mathrm{M}=0.18, \mathrm{SD}=0.17)$.

In order to get a more synthetic view, we calculated a logical score, which was the difference between the mean acceptance rate for all the eight Modus ponens inferences and the mean acceptance rate for all the eight Affirmation of the consequent inferences, and a probability score that was the difference between the mean acceptance rate for the eight high probable conclusions and the mean acceptance rate for the eight low probable conclusions for both the fast and unlimited conditions (see Table 2). Within-subjects t-tests were used to examine the effects of time on each of the scores. The logical score was significantly greater in the unlimited condition than in the fast condition, $\mathrm{t}(54)=8.44, \mathrm{p}<.001$. By contrast, the probability score decreased in the unlimited condition, $\mathrm{t}(54)=$ $3.35, \mathrm{p}<.001$. Nonetheless, the logical score in the fast condition remained significantly greater than $0, \mathrm{t}(54)=6.12, \mathrm{p}<$ .0001 , as did the relative probability score, $\mathrm{t}(54)=5.22$, $\mathrm{p}<$ .0001 .

Despite the very limited time allotted in the fast condition, it is possible that participants might be making certain of these judgments more easily than others, which might explain some of the observed results. In particular, there is evidence that the Modus ponens inference can be made very rapidly (Handley et al., 2011). If this were the case, then we would expect this to be reflected in the pattern of missing responses. Thus, we examined rates of missing responses for the two logical forms (Modus ponens $=28.5 \%$; Affirmation of the consequent $=$ 28.5\%). These were identical and indicate that the observed results were not due to speeded performance on a single logical form.

Overall, as can be seen from Table 2, in the fast condition participants exhibited relatively equal tendencies to make inferences that account for both logical form and relative probability. By contrast, when given unlimited time, there is a clear

Table 2 Logical score and Relative probability score as a function of allotted Time (Fast, Unlimited)

\begin{tabular}{lllll}
\hline Logical score & & & \multicolumn{2}{l}{ Relative probability score } \\
\cline { 5 - 5 } \cline { 5 - 6 } Fast & Unlimited & & Fast & Unlimited \\
\hline $0.36(0.71)$ & $0.71(0.36)$ & & $0.26(0.26)$ & $0.13(0.18)$ \\
\hline
\end{tabular}

increase in the extent to which logical form is used in making inferences and a concomitant decrease in the extent to which relative probability is used.

These results provide a clear replication of the results obtained by Newman et al. (2017), using a somewhat different methodology. They show that when adult participants are given simple inferences with a time that is at the limits of their ability to process the problems, they use a combination of logical form and conclusion probability to make their inferences. By contrast, when given more time, the impact of logical form increases significantly and at the same time there is a decrease in the use of relative probability.

\section{Study $\mathbf{1 b}$}

The results of this initial study provide a useful replication of the effects of restricting time on reasoning with simple familiar premises. We extended this initial analysis in different ways in order to more clearly understand the effects of time, and of the measure used for determining rule-based intuitions. First, we presented participants with a single set of inferences with a single time limit $-15 \mathrm{~s}, 5 \mathrm{~s}$, or $3.5 \mathrm{~s}$. The 15 -s time constraint was chosen to be a real but limited constraint. The 3.5-s constraint was taken from preliminary studies, which indicated that this was close to the shortest time that allowed consistent processing of premises. Second, in line with Newman et al. (2017), we added a set of inferences using abstract premises, in order to provide a second measure of the tendency to make inferences based on logical form. Our main hypothesis here was that the effects of time on tendency to use logical form would be similar for both our initial measure and a measure taken from the abstract problems.

\section{Procedure}

Participants were recruited from an on-line participant pool (Prolific) and were paid $£ 1$ each. Participants were randomly assigned to one of three time conditions $-15 \mathrm{~s}, 5 \mathrm{~s}$, or $3.5 \mathrm{~s}$. Each participant was presented with the same initial instructions as those used in Study 1. They were then presented with 24 inferences randomly presented with a single time limit.

\section{Materials and design}

A set of 24 inferences were randomly presented to each participant. Of these, 16 were identical to those used as the first set of problems used in Study 1. In addition, eight inferences using nonsense terms were also constructed. These presented two Modus ponens inferences and two syllogisms with the form "All $\mathrm{A}$ are $\mathrm{B}, \mathrm{X}$ is $\mathrm{A}$ ", two $\mathrm{AC}$ inferences, and two syllogisms with the form "All A are B, X is B". The following is an example of these: 
All kikinas have boshes in order to plump. Caramel is a kikina. Caramel has boshes in order to plump.

Thus, number of conclusion acceptances was the dependent variable, with Time (15 s, $5 \mathrm{~s}, 3.5 \mathrm{~s})$ as a betweensubjects variable and Logical form (Modus ponens, Affirmation of the consequent), Relative probability (High, Low) and Content (concrete, abstract) as within-subjects variables.

\section{Results and discussion}

We initially analyzed the relation between allotted time and the number of problems for which no response was given in the allotted time. Mean missing responses were 2.76, 4.22, and 4.76 for the $15-\mathrm{s}, 5$-s, and 3.5 -s conditions, respectively. An ANOVA with number of missing responses as dependent variable and Time as independent variable indicated a significant effect of time, $\mathrm{F}(2,147)=4.58, \mathrm{p}<.02$. Contrast analyses indicated that there was a significant increase between $15 \mathrm{~s}$ and $5 \mathrm{~s}$, but no significant differences between $5 \mathrm{~s}$ and 3.5 s.

We then examined performance on the inferential problems. We first analyzed performance on the concrete problems. We performed an ANOVA with rate of conclusion acceptance as dependent variable with Logical form (Modus ponens, Affirmation of the consequent), Relative probability (high, low) and Time ( $15 \mathrm{~s}, 5 \mathrm{~s}, 3.5 \mathrm{~s})$ as repeated measures. This gave significant main effects of logical form, $\mathrm{F}(2,144)=$ $215.39, \mathrm{p}<.001, \eta_{p}{ }^{2}=.599$, and relative probability, $\mathrm{F}(1,144)$ $=31.01, \mathrm{p}<.001, \eta_{p}{ }^{2}=.177$, and significant interactions between time $\times$ logical form, $\mathrm{F}(2,144)=8.45, \mathrm{p}<.001, \eta_{p}{ }^{2}$ $=.105$, and logical form $\times$ plausibility, $\mathrm{F}(1,144)=13.73, \mathrm{p}<$ $.001, \eta_{p}{ }^{2}=.087$.

We then examined patterns of variation in the two ways of assessing rule-based reasoning. We constructed a concrete logical score that was calculated the same way as in Study 1, and an abstract logical score that was the sum of the acceptances of the four Modus ponens forms with abstract content minus the number of acceptances of the four AC forms (see Table 3). We then performed an ANOVA with Logical score as dependent variable with Content (concrete, abstract) as repeated measure and Time ( $15 \mathrm{~s}, 5 \mathrm{~s}, 3.5 \mathrm{~s})$ as dependent variable. This showed significant effects of Time, $\mathrm{F}(2,141)=10.13$, $\mathrm{p}<$ $.001, \eta_{p}^{2}=.126$ and of Content, $\mathrm{F}(1,141)=11.87, \mathrm{p}<.001$,

Table 3 Mean logical score (standard deviations in parentheses) for concrete and abstract inferences as a function of Time (15 s, $5 \mathrm{~s}, 3.5 \mathrm{~s})$

\begin{tabular}{lll}
\hline Time & Concrete logical score & Abstract logical score \\
\hline $15 \mathrm{~s}$ & $2.19(1.72)$ & $1.73(2.09)$ \\
$5 \mathrm{~s}$ & $2.31(1.32)$ & $1.73(1.83)$ \\
$3.5 \mathrm{~s}$ & $1.14(1.58)$ & $0.51(1.92)$ \\
\hline
\end{tabular}

$\eta_{p}{ }^{2}=.077$. The interaction between Content $\times$ Time was not significant, $\mathrm{F}<1$. Thus, although logical scores were somewhat higher with concrete than with abstract content, the pattern of variation by time was the same for both. More specifically, post hoc analyses showed that there was no significant difference in logical scores between $15 \mathrm{~s}$ and $5 \mathrm{~s}$, but that these scores decreased significantly at $3.5 \mathrm{~s}$. Analyses of the logical scores for concrete and abstract content were done using individual $\mathrm{t}$-tests with a Bonferonni correction $(\mathrm{p}=.008)$. This showed that both concrete and abstract logical scores were significantly greater than 0 at $15 \mathrm{~s}$ (concrete: $\mathrm{t}(49)=9.02, \mathrm{p}$ $<.001$, abstract: $\mathrm{t}(48)=5.79, \mathrm{p}<.001)$, and at $5 \mathrm{~s}$ (concrete: $\mathrm{t}(47)=12.10, \mathrm{p}<.001$, abstract: $\mathrm{t}(48)=6.62, \mathrm{p}<.001)$, but at $3.5 \mathrm{~s}$ only the concrete score was significantly greater than 0 , $\mathrm{t}(48)=5.03, \mathrm{p}<.001$, while the abstract score was marginally so: $\mathrm{t}(47)=1.83, \mathrm{p}<.05$. Relative probability scores were similarly analyzed using individual t-tests with a Bonferroni correction $(\mathrm{p}=.016)$. Although there was no significant variation in the relative probability scores by time, these were significantly greater than 0 at all times $(15 \mathrm{~s}: \mathrm{t}(49)=3.31, \mathrm{p}$ $<.001,5 \mathrm{~s}: \mathrm{t}(47)=4.19, \mathrm{p}<.001,3.5 \mathrm{~s}: \mathrm{t}(48)=2.71, \mathrm{p}<.01)$.

These results allow some additional conclusions with respect to the effects of time on measures of use of logical form in reasoning. First, they show that the effects of time were identical for both concrete and abstract logical scores. In combination with the results of Study 1a, they suggest that the ability to use logical form undergoes an initial decrease with the addition of even a moderate level of time constraint, and a second decrease when time is very severely constrained. This is in contrast to relative probability scores, which were not affected by the more severe constraint. The different pattern of time-related change in relative probability and logical scores is also inconsistent with dual-process models that see reasoning as an interplay between two processes.

Second, they show that both concrete and abstract indices appear to track the same basic competence. It is true that logical scores for concrete items are consistently, although very moderately, greater than scores for abstract items. This could be due to a variety of factors - for example, it might be the case that the abstract items are more difficult to process than the concrete items, or conversely that concrete items might provide more cues to logical form. Irrespective, the closeness of the two measures and their parallel pattern of time-related effects suggest that both are equally useful measures of the tendency to use logical form.

\section{Study 2}

These initial studies provide additional evidence that adults have access to a form of rapid reasoning that allows them to consider not only the probabilistic characteristics of potential conclusions but also logical form. Given the short time 
allotted for the fast inferences, this could reasonably be considered to be consistent with the idea that people have a form of basic logical intuition along with a form of rapid reasoning that is sensitive to belief-based information. As previously noted, the results of both the initial studies with adults raise difficulties for the dual-process framework. A further question then concerns the nature of the processes that might underlie the dual nature of fast responding.

As previously noted, there are alternative models that are consistent with these results (Newman et al., 2017). On the one hand, there might be a single underlying process (Kruglanski \& Gigerenzer, 2011; Osman, 2004) that is responsive to the complexity of the corresponding response. This model would suggest that the forms of rule-based responding found in the present study (and those found by Newman et al., 2017) are more complex than the relative probability-based forms of responding, given the way the two change with additional time.

The second model would suggest that there are two relatively autonomous processes that can produce the observed responses. The dual-strategy model of reasoning is one such process (Markovits, Brisson, \& de Chantal, 2017; Markovits et al., 2013; Verschueren et al., 2005). As we have seen, this model postulates the existence of two forms of reasoning strategy. One of these, referred to as a statistical strategy, produces rapid likelihood estimations based on some form of associative access to probabilistic information similar to that postulated by probabilistic theories (Evans et al., 2005; Oaksford \& Chater, 2003; Oaksford \& Chater, 2007). The second, referred to as a counter-example strategy, is more working memoryintensive and requires the generation of a representation of the premises with a corresponding search for potential counterexamples, similar to that postulated by mental model theory (Johnson-Laird \& Byrne, 1991, 1994, 2002). While both strategies can produce responses that are rule-based and beliefbased, statistical strategy use is more sensitive to the latter (Markovits, Brisson, de Chantal, \& Thompson, 2017). Importantly, the previously cited studies show that strategy use is an individual difference, so that reasoners will preferentially use one or the other strategy.

Results obtained with fast and slow responding with adults does not provide any clear way of distinguishing between these models. However, examining the developmental trajectory of fast and slow responding does provide one possible route to doing so. As previously observed, the results obtained with adults indicate that any single process explanation would suggest that rule-based criteria are more complex to generate than are relative probability-based criteria. Given that there is clear evidence that available cognitive resources follow a clear developmental pattern (Gaillard, Barrouillet, Jarrold, \& Camos, 2011; Siegel \& Ryan, 1989), this would suggest that children should show a relative decrease in rule-based responding compared to relative probability-based responding.
Although there have not yet been developmental studies specifically looking at the dual-strategy model, there is evidence suggesting that the two strategies develop in parallel. Markovits and Thompson (2008) found that although probabilistic evaluations are developmentally more precocious, the ability to make counter-example-based judgments starts quite early. There is additional evidence that the ability to distinguish between the Affirmation of the consequent and the Modus ponens inferences follows a clear developmental increase among pre-adolescents (Janveau-Brennan \& Markovits, 1999). These results also indicate that, at the same time, there is an increased sensitivity to belief-based variation (see also De Neys \& Vanderputte, 2011). More recently, supporting neurological evidence for such a parallel development in children has been found (Schwartz, Epinat-Duclos, Léone, \& Prado, 2017). This evidence strongly suggests that there is a parallel developmental trajectory for both probability-based and counter-example-based strategies. If this is, indeed, the case, then the developmental pattern should show a more even developmental trajectory for rapid and slow rule-based and belief-based responding.

We thus replicated the initial study with children in Grades 4 and 6 (with ages ranging between 8 and 10 years of age). We gave children the concrete items used in Studies $1 \mathrm{a}$ and $1 \mathrm{~b}$, since these parallel results obtained with abstract items, and it was felt that children would have difficulty in processing abstract items. Previous studies have shown that the ability to perform the simple kinds of reasoning tasks examined here show a clear developmental increase in this age range, with older children performing fairly similarly to adults (JanveauBrennan \& Markovits, 1999; Markovits, 2017).

We made one modification in the methodology. In order to accommodate possible variations in children's reading abilities, we devised a procedure that allowed some estimation of this parameter. We gave children an initial series of five statement sets that were approximately the same length as the inferences that were to be presented. The initial set of statements was presented in $5 \mathrm{~s}$ and each successive inference was presented for a time that increased by approximately $1 \mathrm{~s}$ from the previous one. Children were simply asked to indicate by clicking a button if they had the time to read the statements. The fastest time that was thus indicated was used as the time in the initial fast condition.

\section{Pretest}

Although the relative probability conclusions used in these initial studies were chosen to have a strong level of face validity, we considered it to be useful to verify this with children. We accordingly presented a series of dichotomous choices to 19 children who were in Grade 5 ( 10 years, 3 months; nine girls). Each of the choices presented the high- and low-relative probability conclusions for the same premises that were used in the 32 problems 
used in Study 1. For each choice, children were asked to indicate which of the two statements was most likely to occur. This gave a total of 16 dyadic comparisons. Children were given 1 point each time that they chose the high-relative probability statement. Mean score was 14.4 , which indicated a very strong tendency to choose the high-relative frequency statement as being more probable, $\mathrm{t}(18)=22.91, \mathrm{p}<.0001$.

\section{Participants}

A total of 54 children participated in this study. Of these, 38 were in Grade 6 ( 21 girls; 17 boys: mean age $=11$ years, 1 month) and 21 were in Grade 4 (ten girls, 11 boys: mean age $=$ 9 years, 0 months). Actual sample size varied as a function of class size and permissions, but we aimed for a minimum of 20 subjects per age group, which gave a lower bound of a $70 \%$ chance of detecting a medium effect size.

\section{Materials and design}

A computer program was created using Microsoft Visual Basic. The opening screen briefly described the study and informed participants that they could stop responding at any time with no consequence. Following this, participants were told that they would be asked to read a series of statements on the screen, and that each be presented for a very brief period of time. They were asked to click on a button below each set of statements if they had been able to read the statements. The first set of statements was presented for $5 \mathrm{~s}$. Each subsequent statement was presented for a time that was $1 \mathrm{~s}$ more than the previous one.

Following this, participants were given the exact same protocol as was used with the adults in Study 1.

Thus, number of conclusion acceptances was the dependent variable with Time (Fast, Unlimited) as a betweensubjects variable and Logical form (Modus ponens, Affirmation of the consequent) and Relative probability (High, Low) as within-subjects variables.

\section{Procedure}

Participants were seen in groups of five in a quiet room and did the experiment on a portable computer.

\section{Results and discussion}

We first analyzed the time taken to read the initial set of statements. The average time used in the fast condition was $6.7 \mathrm{~s}$ in Grade 4 and $6.1 \mathrm{~s}$ in Grade 6. The mean number of nonresponses was $47.5 \%$ at Grade 4 and $39.2 \%$ at Grade 6 . As in the previous study, we eliminated participants who failed to answer at least one problem in each of the four categories. This left a total of 18 participants in Grade 4 and 36 participants in Grade 6.
We then performed an ANOVA with Rate of conclusion acceptance as dependent variable with Logical form (MP, AC), Relative probability (High, Low), and Time (Fast, Unlimited) as repeated measures and Grade $(4,6)$ as a between-subjects variable. This gave significant main effects of Logical form, $\mathrm{F}(1,52)=18.21, \mathrm{p}<.001, \eta_{p}{ }^{2}=.262$, Relative probability, $\mathrm{F}(1,52)=52.52, \mathrm{p}<.001, \eta_{p}{ }^{2}=.502$, Time, $\mathrm{F}(1,52)=6.22, \mathrm{p}$ $<.04, \eta_{p}{ }^{2}=.107$, and significant interactions involving Time $\times$ Grade, $\mathrm{F}(1,52)=4.92, \mathrm{p}<.03, \eta_{p}{ }^{2}=.086$, Relative probability $\times$ Grade, $\mathrm{F}(1,52)=5.51, \mathrm{p}<.02, \eta_{p}{ }^{2}=.096$, Time $\times$ Logical form, $\mathrm{F}(1,52)=9.10, \mathrm{p}<.005, \eta_{p}{ }^{2}=.149$, Time $\times$ Relative probability, $\mathrm{F}(1,52)=12.75, \mathrm{p}<.001, \eta_{p}{ }^{2}=.197$, and Time $\times$ Logical form $\times$ Relative probability $\times$ Grade, $\mathrm{F}(1,52)=6.76, \mathrm{p}<.02, \eta_{p}{ }^{2}=.115$.

Overall, the Modus ponens inference was accepted more often $(\mathrm{M}=0.73, \mathrm{SD}=0.21)$ than the Affirmation of the consequent inference $(M=0.55, S D=0.27)$. High probable inferences were accepted more often $(\mathrm{M}=0.74, \mathrm{SD}=0.20)$ than were low probable inferences $(\mathrm{M}=0.54, \mathrm{SD}=0.26)$. The rate of acceptance of inferences in the fast condition $(\mathrm{M}=0.68$, SD $=0.23)$ was greater than in the unlimited condition $(\mathrm{M}=0.59$, SD 0.23). Post hoc analyses of the interactions were done using the Tukey test with $\mathrm{p}=.05$. Analysis of the Grade $\times$ Time interaction did not indicate any significant differences. Analysis of the Grade $\times$ Relative probability interaction showed that in Grade 6 the rate of acceptance of the high probable inferences $(\mathrm{M}=0.73, \mathrm{SD}=0.18)$ was significantly greater than the rate of acceptance of the low probable inferences $(M=0.49, S D=0.26)$, while this difference was not significant in Grade 4 (high probable $=0.75, \mathrm{SD}=0.23$, low probable $=0.63, \mathrm{SD}=0.23$ ). Analysis of the Time $\times$ Logical form interaction showed that there was no difference in acceptance rates for the Modus ponens inference in the fast $(\mathrm{M}=$ $0.74, \mathrm{SD}=0.24)$ than in the unlimited condition $(\mathrm{M}=0.71$, $\mathrm{SD}=0.24$ ), while the Affirmation of the consequent inference was accepted more often in the fast condition $(\mathrm{M}=0.63, \mathrm{SD}=$ $0.29)$ than in the unlimited condition $(\mathrm{M}=0.47, \mathrm{SD}=0.32)$. Analysis of the Time $\times$ Relative probability interaction indicated that the rate of acceptance of the high probable items was similar in the fast condition $(\mathrm{M}=0.75, \mathrm{SD}=0.22)$ than in the unlimited condition $(\mathrm{M}=0.72, \mathrm{SD}=0.23)$, while the rate of acceptance of the low probable items was greater in the fast condition $(\mathrm{M}=0.62, \mathrm{SD}=0.30)$ than in the unlimited condition $(\mathrm{M}=0.45, \mathrm{SD}=0.20)$. Analysis of the four-way interaction did not show specific significant differences between Grade levels (see Table 4), and was not further analyzed.

As for the adults, we calculated a logical score and a relative probability score (see Table 5). We then conducted separate ANOVAs for both the logical and the probability score with Time as a repeated measure and Grade as a between-subjects variable. For the logical score, this showed significant effects of time, $\mathrm{F}(1,52)=9.10, \mathrm{p}<.001, \eta_{p}{ }^{2}=.149$, and Grade, $\mathrm{F}(1,52)=$ $3.90, \mathrm{p}=.05, \eta_{p}{ }^{2}=.070$. Overall, the logical score was greater at Grade 6 than at Grade 4. The logical score was greater in the 
Table 4 Proportion of conclusion acceptance (standard deviations in parentheses) as a function of Form (Modus ponens (MP), Affirmation of the consequent (AC)), level of Relative probability (High, Low) and Grade level $(4,6)$ as a function of allotted Time (Fast, Unlimited)

\begin{tabular}{|c|c|c|c|c|c|}
\hline \multirow[t]{2}{*}{ Grade } & \multirow[t]{2}{*}{ Time } & \multicolumn{2}{|l|}{ MP } & \multicolumn{2}{|l|}{$\mathrm{AC}$} \\
\hline & & High & Low & High & Low \\
\hline \multirow[t]{2}{*}{4} & Fast & $0.74(0.28)$ & $0.67(0.31)$ & $0.67(0.31)$ & $0.52(0.27)$ \\
\hline & Unlimited & $0.84(0.27)$ & $0.66(0.36)$ & $0.75(0.28)$ & $0.48(0.32)$ \\
\hline \multirow[t]{2}{*}{6} & Fast & $0.84(0.20)$ & $0.66(0.33)$ & $0.70(0.27)$ & $0.41(0.40)$ \\
\hline & Unlimited & $0.90(0.20)$ & $0.48(0.35)$ & $0.49(0.36)$ & $0.30(0.33)$ \\
\hline
\end{tabular}

unlimited condition than in the fast condition. For the relative probability score, this also showed significant effects of time, $\mathrm{F}(1,52)=12.5, \mathrm{p}<.001, \eta_{p}{ }^{2}=.197$, and Grade, $\mathrm{F}(1,52)=$ $5.51, \mathrm{p}<.03, \eta_{p}{ }^{2}=.096$. The probability score varied in the same way as the logical score as a function of Grade and time.

We then examined whether the logical and relative probability scores were significantly greater than 0 in the limited time condition. For the Grade 6 children, both the logical score, $\mathrm{t}(35)=3.01$, $\mathrm{p}<.01$ and the relative probability score, $\mathrm{t}(35)=4.57, \mathrm{p}<.001$, were significantly greater than 0 , while for the Grade 4 children neither scores were significantly greater than 0 ( $t<1$ for both).

One result that merits some additional examination is the very low level of both logical and relative probability scores among the grade 4 children. Although these scores are consistent with the general pattern, it is possible that some of these children misunderstood the instructions for the initial statement set and were not able to read the fast problems adequately, thus underestimating the real level. In order to examine this, we looked at the 13 Grade 4 children who were allotted over $6.5 \mathrm{~s}$ to do the fast condition. For these participants, both the logical score $(\mathrm{M}=0.15)$ and the relative probability score $(\mathrm{M}=0.03)$ remained very low.

Finally, as in the previous study, we examined rates of nonresponding for the Modus ponens and Affirmation of the consequent inferences for each grade. These showed very similar rates for both Grade 4 (Modus ponens $=46.2 \%$, Affirmation of the consequent $=48.7 \%$ ) and Grade 6 (Modus ponens $=38.8 \%$, Affirmation of the consequent $=39.5 \%$ ).

Overall, these results show that fast responding reflects both the influence of logical form and probability at Grade 6 , although the extent to which this was the case was less than

Table 5 Logical score and Relative probability score as a function of allotted Time (Fast, Unlimited) and Grade $(4,6)$

\begin{tabular}{llllll}
\hline Grade & \multicolumn{2}{l}{ Logical score } & & \multicolumn{2}{l}{ Relative probability score } \\
\cline { 2 - 3 } \cline { 6 - 6 } \cline { 5 - 6 } & Fast & Unlimited & & Fast & Unlimited \\
\hline 4 & $0.03(0.24)$ & $0.14(0.23)$ & & $0.03(0.20)$ & $0.22(0.27)$ \\
6 & $0.14(0.28)$ & $0.30(0.36)$ & & $0.18(0.24)$ & $0.30(0.22)$ \\
\hline
\end{tabular}

found with the adults. For the Grade 4 children, there was no evidence of either logical form or probability being used to make rapid inferences. In both cases, the unlimited time condition produced clear increases in the extent to which both logical form and relative probability were used as inferential cues.

\section{Discussion}

Previous results obtained by Newman et al. (2017) provide direct evidence that when adults make very rapid conditional inferences, these use a combination of both rule-based and beliefbased cues. The authors conclude that their results are inconsistent with typical dual-process frameworks. The results of the present studies with adults both confirm and extend these using a different methodology. In particular, results showing a different time course of change in the use of rule-based and belief-based cues with adults also poses additional difficulties for dual-process theories. More importantly, results examining the developmental pattern change in slow and fast responding provide even stronger evidence for this general conclusion. In these studies, both the Grade 6 children and the adults show fast responding that clearly indicates use of a combination of both logical form and relative probability as cues to validity, although there is little evidence of this in the Grade 4 children. There are, in addition, some important developmental patterns, which are summarized in Table 6 . First, there is a consistent developmental increase in the relative use of both logical form and relative probability in fast responding that extends from the youngest children to adults. Second, both for the Logical and for the Relative probability scores there is a great similarity between fast responding at a given grade level and unlimited responding at the previous developmental level.

These results have some clear implications. First, the overall patterns of responding allow some modulation of one of the major explanations of the effects of having limited cognitive resources on the interaction between rule-based and beliefbased responding. This suggests that limited cognitive resources increase use of Type 1 reasoning and concomitantly decrease use of Type 2 reasoning. The results found with the adults in the present study are indeed consistent with those of other studies (De Neys, 2006; Evans \& Curtis-Holmes, 2005) since they show a decrease in the use of logical form and increased use of relative probability in the fast compared to the unlimited condition. However, examination of the results of the Grade 4 and the Grade 6 students shows a very different pattern. In this case, fast responding shows a relative decrease in the use of both logical form and relative probability. This is mirrored by the developmental pattern of responding in the unlimited condition. The older children show clear increases in the extent to which both logical form and relative probability are used as cues to validity. 
Table 6 Logical score and Relative probability score in the fast condition for each grade level and in the unlimited condition for the previous grade level

\begin{tabular}{llllll}
\hline Grade & \multicolumn{2}{l}{ Logical score } & & \multicolumn{2}{l}{ Relative probability score } \\
\cline { 2 - 3 } & Previous unlimited & Fast & & Previous unlimited & Fast \\
\hline 4 & & $0.03(0.24)$ & & $0.03(0.20)$ \\
6 & $0.14(0.23)$ & $0.14(0.28)$ & & $0.22(0.27)$ & $0.18(0.24)$ \\
University & $0.30(0.36)$ & $0.36(0.71)$ & & $0.30(0.22)$ & $0.26(0.26)$ \\
\hline
\end{tabular}

This parallel developmental pattern is difficult to explain by a single-process model. Any such explanation that relies on a single dimension such as complexity (e.g., Kruglanski \& Gigerenzer, 2011; Osman, 2004) would suggest an asymmetrical pattern of change in which time constraint and developmental level would have a stronger effect on belief-based cues. This problem also applies to other dimensions such as children's understanding of "logical" instructions, which should also have an asymmetrical effect.

These results are also disconcerting for many dual-process theories, especially since Type 1 processes are generally considered to be more primitive than Type 2 processes (Evans \& Stanovich, 2013). Under any reasonable interpretation of the nature of Type 1 processes, one would expect these to be developmentally precocious. However, the pattern of responding among the younger participants indicates rather that the processes responsible for both logical and knowledge-based responses appear to develop in parallel, as indeed is suggested by the dual-strategy model.

The second important part of these results is the similarity between fast responding and limited time responding at the previous development level. The simplest explanation of this would rely on the notion of cognitive resources. Restricting the time allotted to make an inference is one way of reducing available cognitive resources. In addition, one of the clearest and most well documented developmental differences is the gradual increase in available cognitive resources (Gaillard et al., 2011; Siegel \& Ryan, 1989). This is, as an example, the major premise in Barrouillet's developmental explanation of differences in reasoning (Barrouillet \& Lecas, 1999; Gauffroy \& Barrouillet, 2009). Thus, we can understand the similarity between fast responding at a given developmental level and unlimited responding at a previous developmental level as mirroring the effects of the relative availability of cognitive resources. What this suggests, then, is that the results with the children and with the adults in the fast condition are consistent with the idea that the processes responsible for use of logical cues and knowledge-based cues work in parallel, both developmentally and under time constraints.

It is, however, possible that the developmental patterns observed here might be explained by factors other than the underlying processes at play. One possibility is that the children and the adults do not understand the instructions in the same way, with younger children interpreting instructions as asking for some measure of plausibility, rather than logical validity. However, if this were the case, then one would expect the difference between use of logical and relative probability cues to diverge in a pattern that would be opposite to that found with the adults, which is not the case. In addition, since any such problem would be stronger among the younger children, we would expect that the relative use of rule-based criteria compared to relative probability criteria would increase among the older children, which is also not the case. Another possibility is that there is a developmental modification in the nature of Type 1 and Type 2 processes. However, while this is possible, it would involve a major change in the way that dual-process theories are construed.

By contrast, the dual-strategy model of reasoning (Markovits, Brisson, \& de Chantal, 2017; Markovits et al., 2013; Verschueren et al., 2005) provides a useful basis for explaining this pattern of results. This model proposes the co-existence of two modes of inferential reasoning, a statistical strategy that uses implicit statistical (i.e., knowledge-based) information in order to produce an intuitive estimate of conclusion likelihood, and a counterexample strategy that uses the same basis in order to determine whether or not there exist potential counter-examples for a given conclusion. The developmental results in the current study are completely consistent with results indicating that probabilitybased inferences and counter-example-based inferences show parallel developmental patterns (Janveau-Brennan \& Markovits, 1999; Markovits \& Thompson, 2008; Schwartz et al., 2017). Results showing that fast responding in adults is very similar to slow responding in the older children is particularly telling in this respect.

However, irrespective of the specific interpretation, these results show that fast responding in both children and adults clearly reflects the same developmental pattern, with both logical intuitions and knowledge-based intuitions coexisting in an increasingly effective way. In addition, they show that these intuitions follow the developmental pattern of slow responding, giving strong evidence that they are the result of an experiential process.

Acknowledgements This study was supported by a Discovery grant from the Natural Sciences and Engineering Research Council of Canada to HM.

Open Practices Statement The data and materials for all experiments are available on demand and none of the experiments was preregistered. 


\section{Appendix}

\section{Problem Set A}

If there is lightning, then there is noise.

There is lightning.

Conclusion: there is noise.

If someone falls in a river, then they will drown.

Jean fell in a river.

Jean drowned.

If a candle is lit, then there will be light.

A candle is lit.

There is light.

If an object is put into a fire, it will explode.

An object is put into a fire.

The object exploded.

All dogs have lungs in order to breathe.

Caramel is a dog.

Caramel has lungs in order to breathe.

All birds swim in water.

Cacahuette is a bird.

Cacahuette swims in water.

All coffee has caffeine.

This drink is a coffee.

This drink has caffeine.

All trains work on steam.

This is a train.

It works on steam.

If a ball is thrown in a window, then the window will break. AC high

The window is broken.

A ball was thrown in the window.

If there is a banana skin on the sidewalk, it is slippery.

The sidewalk is slippery.

There is a banana skin on the sidewalk.

If a fire is lit, then there is smoke.

There is smoke.

A fire was lit.

If a person is hosed by a fireman, then they will be wet.

A person is wet.

The person was hosed by a fireman.

All dogs are domestic animals.

Riki is a domestic animal.

Riki is a dog.

All airplanes serve alcoholic beverages.

A place serves alcoholic beverages.

This place is an airplane.

All schools offer courses.

A Place offers courses.

This place is a school.

All lawnmowers have a motor.

This object has a motor.

Object is a lawnmower.
MP high

MP high

MP low

MP high

MP low

MP low

AC low

C high

AC low

AC low

AC low

\section{Problem Set B}

If there is lightning, then there is a forest fire.

There is lightning.

Conclusion: there is a forest fire.

If someone falls in a river, then they will be wet.

Jean fell in a river.

Jean is wet.

If a candle is lit, then there will be a party.

A candle is lit.

There is a party.

If an object is put into a fire, it will be hot.

An object is put into a fire.

The object is hot.

All dogs are guides for blind people.

Caramel is a dog.

Caramel is a guide for blind people.

All birds fly in the air.

Castello is a bird.

Castello flies in the air.

All coffee is Indonesian.

This drink is a coffee.

This drink is Indonesian.

All trains travel on rails.

This is a train.

It travels on rails.

If a sofa is thrown in a window, then the window will break. AC low

The window is broken.

A sofa was thrown in the window.

If there is place on the sidewalk, it is slippery. AC high

The sidewalk is slippery.

There is ice on the sidewalk.

If there is a factory, then there is smoke.

AC low

There is smoke.

There is a factory.

If a person walks in the rain, then they will be wet.

AC high

A person is wet.

The person walked in the rain.

All snakes are domestic animals.

AC low

Tofu is a domestic animal.

Tofu is a snake.

All bars serve alcoholic beverages.

AC high

A place serves alcoholic beverages.

This place is a bar.

All shooting clubs offer courses.

AC low

A Place offers courses.

This place is a shooting club.

All cars have a motor

This object has a motor.

This object is a car. 


\section{References}

Barrouillet, P., \& Lecas, J.-F. (1999). Mental models in conditional reasoning and working memory. Thinking \& Reasoning, 5(4), 289-302.

De Neys, W. (2006). Dual processing in reasoning: Two systems but one reasoner. Psychological Science, 17(5), 428-433.

De Neys, W. (2012). Bias and conflict a case for logical intuitions. Perspectives on Psychological Science, 7(1), 28-38.

De Neys, W., \& Glumicic, T. (2008). Conflict monitoring in dual process theories of thinking. Cognition, 106(3), 1248-1299.

De Neys, W., \& Vanderputte, K. (2011). When less is not always more: Stereotype knowledge and reasoning development. Developmental Psychology, 47(2), 432.

De Neys, W., Vartanian, O., \& Goel, V. (2008). Smarter than we think: When our brains detect that we are biased. Psychological Science, 19(5), 483-489.

Epstein, S. (1994). Integration of the cognitive and the psychodynamic unconscious. American Psychologist, 49(8), 709.

Evans, J. S. B. (2006). The heuristic-analytic theory of reasoning: Extension and evaluation. Psychonomic Bulletin \& Review, 13(3), 378-395.

Evans, J. S. B., Curtis-Holmes, J. (2005). Rapid responding increases belief bias: Evidence for the dual-process theory of reasoning. Thinking \& Reasoning, 11(4), 382-389.

Evans, J. S. B., Over, D. E., \& Handley, S. J. (2005). Suppositionals, extensionality, and conditionals: A critique of the mental model theory of Johnson-Laird and Byrne (2002). Psychological Review, 112, 1040-1052.

Evans, J. S. B., \& Stanovich, K. E. (2013). Dual-process theories of higher cognition advancing the debate. Perspectives on Psychological Science, 8(3), 223-241.

Frederick, S. (2005). Cognitive reflection and decision making. Journal of Economic Perspectives, 19(4), 25-42.

Gaillard, V., Barrouillet, P., Jarrold, C., \& Camos, V. (2011). Developmental differences in working memory: Where do they come from? Journal of Experimental Child Psychology, 110(3), 469-479.

Gauffroy, C., \& Barrouillet, P. (2009). Heuristic and analytic processes in mental models for conditionals: An integrative developmental theory. Developmental Review, 29(4), 249-282.

Handley, S. J., Newstead, S. E., \& Trippas, D. (2011). Logic, beliefs, and instruction: A test of the default interventionist account of belief bias. Journal of Experimental Psychology: Learning, Memory, and Cognition, 37(1), 28.

Janveau-Brennan, G., Markovits, H. (1999). The development of reasoning with causal conditionals. Developmental Psychology, 35(4), 904-911.

Johnson-Laird, P. N., \& Byrne, R. M. (1994). Models, necessity, and the search for counter-examples. Behavioral and Brain Sciences, 17(4), 775-777.

Johnson-Laird, P. N., \& Byrne, R. M. J. . (1991). Deduction. Hove \& London: Erlbaum.

Johnson-Laird, P. N., \& Byrne, R. M. J. (2002). Conditionals: A theory of meaning, pragmatics and inference. Psychological Review, 109, 646-678.

Kahneman, D. (2011). Thinking, fast and slow: Macmillan, London.

Kruglanski, A. W., \& Gigerenzer, G. (2011). Intuitive and deliberate judgments are based on common principles. Psychological Review, 118(1), 97.
Markovits, H. (2017). In the beginning stages: Conditional reasoning with category based and causal premises in 8-to 10 -year olds. Cognitive Development, 41, 1-9.

Markovits, H., Brisson, J., \& de Chantal, P.-L. (2017). Logical reasoning versus information processing in the dual-strategy model of reasoning. Journal of Experimental Psychology: Learning, Memory, and Cognition, 43(1), 72.

Markovits, H., Brisson, J., de Chantal, P.-L., \& Thompson, V. A. (2017). Interactions between inferential strategies and belief bias. Memory \& Cognition, 1-11.

Markovits, H., Brunet, M.-L., Thompson, V., \& Brisson, J. (2013). Direct evidence for a dual process model of deductive inference. Journal of Experimental Psychology: Learning, Memory, and Cognition, 49(6), 1213-1222.

Markovits, H. Thompson, V. (2008). Different developmental patterns of simple deductive and probabilistic inferential reasoning. . Memory and Cognition, 36(6), 1066-1078.

Markovits, H., Trémolière, B., \& Blanchette, I. (2018). Reasoning strategies modulate gender differences in emotion processing. Cognition, 170, 76-83.

Morsanyi, K., \& Handley, S. J. (2012). Logic feels so good-I like it! Evidence for intuitive detection of logicality in syllogistic reasoning. Journal of Experimental Psychology-Learning Memory and Cognition, 38(3), 596.

Newman, I. R., Gibb, M., \& Thompson, V. A. (2017). Rule-based reasoning is fast and belief-based reasoning can be slow: Challenging current explanations of belief-bias and base-rate neglect. Journal of Experimental Psychology: Learning, Memory, and Cognition, 43(7), 1154.

Oaksford, M., \& Chater, N. (2003). Conditional probability and the cognitive science of conditional reasoning. Mind \& Language, 18(4), 359-379.

Oaksford, M. \& Chater, N. (2007). Baysian rationality. Oxford, England: Oxford University Press.

Osman, M. (2004). An evaluation of dual-process theories of reasoning. Psychonomic Bulletin \& Review, 11(6), 988-1010.

Pennycook, G., Fugelsang, J. A., \& Koehler, D. J. (2012). Are we good at detecting conflict during reasoning? Cognition, 124(1), 101-106.

Quinn, S., \& Markovits, H. (1998). Conditional reasoning, causality, and the structure of semantic memory: Strength of association as a predictive factor for content effects. Cognition, 68(3), B93-B101.

Schwartz, F., Epinat-Duclos, J., Léone, J., \& Prado, J. (2017). The neural development of conditional reasoning in children: Different mechanisms for assessing the logical validity and likelihood of conclusions. Neuroimage, 163, 264-275.

Siegel, L. S., \& Ryan, E. B. (1989). The development of working memory in normally achieving and subtypes of learning disabled children. Child Development, 973-980.

Simon, G., Lubin, A., Houdé, O., \& De Neys, W. (2015). Anterior cingulate cortex and intuitive bias detection during number conservation. Cognitive Neuroscience, 6(4), 158-168.

Sloman, S. A. (1996). The empirical case for two systems of reasoning. Psychological Bulletin, 119(1), 3-22.

Stanovich, K. E. (1999). Who is rational?: Studies of individual differences in reasoning. Mahwah, NJ: Lawrence Erlbaum Associates Publishers.

Stanovich, K. E., \& Toplak, M. E. (2012). Defining features versus incidental correlates of Type 1 and Type 2 processing. Mind \& Society, 11(1), 3-13. 
Thompson, V. A., Prowse Turner, Jamie A., Pennycook, G. (2011). Intuition, reason, and metacognition. Cognitive Psychology, 63, 107-140.

Toplak, M. E., West, R. F., \& Stanovich, K. E. (2011). The Cognitive Reflection Test as a predictor of performance on heuristics-andbiases tasks. Memory \& Cognition, 39(7), 1275.

Verschueren, N., Schaeken, W., \& d'Ydewalle, G. (2005). Everyday conditional reasoning: A working memory- dependent tradeoff between counter-example and likelihood use. Memory \& Cognition, 33(1), 107-119.

Publisher's note Springer Nature remains neutral with regard to jurisdictional claims in published maps and institutional affiliations. 